flare cluster included Delaware, Delaware Bay area, and Chesapeake Bay area between 2003 and 2014. Maps were generated highlighting the study area, flares, and identified clusters from all analyses. The space-time effects of environmental and demographic variables on the identified clusters will be considered in subsequent analysis.

Conclusions We describe the first space-time clusters of lupus organ-specific disease activity strongly supporting the role of environmental factors as drivers of lupus activity.

\section{PS3:46 RELATIONSHIP BETWEEN DAMAGE CLUSTERING AND MORTALITY IN JUVENILE SYSTEMIC LUPUS ERYTHEMATOSUS: CLUSTER ANALYSES IN A LARGE COHORT FROM THE SPANISH SOCIETY OF RHEUMATOLOGY LUPUS REGISTRY}

${ }^{1} \mathrm{~V}$ Torrente-Segarra, ${ }^{2} \mathrm{TC}$ Salman-Monte, ${ }^{3} \mathrm{R}$ Rua-Figueroa, ${ }^{4} \mathrm{~J}$ Calvo-Alén ${ }^{5} \mathrm{FJ}$ Jópez-longo, ${ }^{6} \mathrm{M}$ Galindo, ${ }^{7} \mathrm{~A}$ Olivé, ${ }^{8} \mathrm{JM}$ Pego-Reigosa. ${ }^{1}$ Hospital General Hospitalet-HSJDMoisès Broggi, Universitat de Barcelona (UB), Hospitalet Llobregat, Spain; ${ }^{2}$ Parc de Salut Mar-IMIM, Department of Medicine, Universitat Autònoma de Barcelona (UAB), Barcelona, Spain; ${ }^{3}$ Hospital Universitario Dr Negrín, Las Palmas de Gran Canaria, Spain; ${ }^{4}$ Hospital Cruces, Bilbao, Spain; ${ }^{5}$ Hospital Gregorio Marañón, Madrid, Spain; ${ }^{6}$ Instituto de Investigación Hospital 12 de Octubre (I+12), Madrid, Spain; ' ${ }^{7}$ Hospital Germans Tries i Pujol, Badalona, Spain; ${ }^{8}$ Hospital do Meixoeiro, Vigo, Spain

\subsection{6/lupus-2018-abstract.94}

Objectives To identify patterns (clusters) of damage manifestations within a large cohort of juvenile SLE (jSLE) patients and evaluate the potential association of these clusters with a higher risk of mortality.

Methods This is a multicentre, descriptive, cross-sectional study of a cohort of 345 jSLE patients from the Spanish Society of Rheumatology Lupus Registry. Organ damage was ascertained using the Systemic Lupus International Collaborating Clinics Damage Index. Using cluster analysis, groups of patients with similar patterns of damage manifestations were identified.

Results Mean age at diagnosis 14.2 2 2.89, $88.7 \%$ were female and $93.4 \%$ were Caucasian. A total of 12 (3.5\%) patients died, mean SLICC/ACR DI 1.27 \pm 1.63 . Three damage clusters were identified:

Cluster $1(72.7 \%$ of patients) showed damage in only $22.3 \%$ of patient, but no significant domain was involved.

Cluster $2(14.5 \%)$ was featured by renal damage in $60 \%$ of patients, ocular damage in 54\%, cardiovascular damage in $20 \%$ and gonadal failure in $14 \%$, all significantly higher than clusters 1 and $3(\mathrm{p}<0.001)$. All patients scored for some damage in SLICC/ACR DI index, with a mean of $2.90 \pm 1.54$ and mean affected domains of $1.86 \pm 0.93$.

Cluster $3(12.7 \%)$ was the only group with musculoskeletal damage (100\%), clearly higher than clusters 1 and 2. All patients scored for some damage in SLICC/ACR DI index, with a mean of $2.66 \pm 1.87$ and mean affected domains of $1.89 \pm 1.18$.

The overall mortality rate of patients in clusters 2 and 3 was higher than in cluster $1(\mathrm{p}<0.05)$ and significantly higher in cluster $2(2.2 \mathrm{x}$ times than cluster 3 and $5 \mathrm{x}$ times than cluster 1) (See table 1).

Conclusion In a large cohort of jSLE patients, we found one cluster with several damage domains involved that we consider clinically meaningful. Another cluster with important musculoskeletal damage manifestations and another cluster with no clinically significant damage at all were also found. These two clusters of jSLE with important clinical damage were found to be associated to higher rates of mortality, specially for the cluster involving renal, ocular, cardiovascular and gonadal domains. Physicians should pay special attention to the early prevention of damage in these particular subsets of patients.

\section{PS3:47 MULTI-YEAR ANALYSIS OF PREVALENCE/OUTCOMES OF PULMONARY EMBOLISM IN SYSTEMIC LUPUS ERYTHEMATOSUS DISCHARGES FROM NATIONWIDE INPATIENT SAMPLE DATABASE \& COMPARISON TO NATIONAL HOSPITAL DISCHARGE SURVEY}

V Majithia, T Nasir, S Lirette, S Kishore. University of Mississippi School of Medicine, Jackson, USA

10.1136/lupus-2018-abstract.95

Abstract PS3:47 Table 1 Outcomes

\begin{tabular}{|l|c|c|c|c|c|}
\hline Variable & All Patients & PE & No PE & OR & p-Value \\
\hline Inpatient Mortality & $6132(2 \%)$ & $226(6 \%)$ & $5906(2 \%)$ & $2.99(\mathrm{Cl} 2.56-3.49)$ & $<0.001$ \\
\hline $\begin{array}{l}\text { Moderate to severe } \\
\text { disability at discharge }\end{array}$ & $77904(26 \%)$ & $1184(31 \%)$ & $76720(26 \%)$ & $1.26(\mathrm{Cl} 1.16-1.37)$ & $<0.001$ \\
\hline $\begin{array}{l}\text { Variable } \\
\text { LOS Median (IQR), Days }\end{array}$ & All Patients & PE & No PE & Co-efficient & p-Value \\
\hline $\begin{array}{l}\text { Total Charges (\$10,000) } \\
\text { Median (IQR) }\end{array}$ & 23100 & 34900 & 22900 & $1.94(\mathrm{Cl} 1.59-2.29)$ & $<0.001$ \\
\hline
\end{tabular}

Data are presented as Number of patients (\%) or Median ( \pm IQR).

Abbreviations: OR = Odds Ratio ( $95 \%$ Confidence Intervals $(\mathrm{CI})$ ); LOS = Length of stay; PE = Pulmonary Embolism; IQR = Interquartile range 
Abstract PS3:47 Table 2 Comparison of results between NIS and NHDS databases

\begin{tabular}{|l|c|c|}
\hline \multicolumn{1}{|c|}{ Characteristics and outcomes in SLE with PE } & NIS & NHDS \\
\hline Mean age & 49 years & 47.9 years \\
\hline Overall number of Females & $\mathbf{8 6 \%}$ & $90.4 \%$ \\
\hline Risk of developing PE with SLE & $\mathbf{1 . 8 4 *}$ & $\mathbf{2 . 0}$ \\
\hline Prevalence in African-Americans vs Caucasians & $1.5 \%$ vs $1.2 \%$ & $1.9 \%$ vs $1.7 \%$ \\
\hline Length of stay (Days) & 6 & 6.7 \\
\hline Increase in Inpatient mortality & $\mathbf{3}$ times * & $\mathbf{1 . 2 1}$ times * \\
\hline Prevalence in Males vs Females & $1.7 \%$ vs $1.2 \%$ & $1.3 \%$ vs $1.7 \%$ \\
\hline & \multicolumn{2}{|c}{$* \mathbf{*}$ p-value $<\mathbf{0 . 0 0 0 1}$} \\
\hline
\end{tabular}

\section{Abbreviations: Nationwide Inpatient Sample database (NIS); National Hospital Discharge Survey (NHDS); Systemic lupus erythematosus (SLE); Pulmonary Embolism (PE)}

Background Systemic Lupus Erythematosus (SLE) may increase this risk of acute pulmonary embolism (PE) and its complications in hospitalised patients. Herein, Nationwide Inpatient Sample (NIS) database from 2003-2011 was analysed to assess the relationship of PE and SLE in hospitalised patients.

Methods NIS database (2003-2011) was queried to identify all adults (age $>18$ years) with SLE and PE using appropriate ICD-9 codes. Demographic characteristics and in-hospital outcomes were compared between SLE patients with and without a PE. Multivariate logistic regression analysis was used to obtain adjusted odds ratio (OR).

Results Of 299595 hospitalizations of patients with SLE from 2003-2011, 3839 (1.28\%) had PE, 1.83 times of the background prevalence with OR $1.85(\mathrm{p}<0.0001)$. Overall, $89 \%$ were females with mean age of 51 years, while those with PE were younger, mean age of 49 years. Rate of PE was higher in African-Americans vs Caucasians (1.5\% vs $1.2 \%)$ and in males vs females $(1.7 \%$ vs $1.2 \%)$. After adjusting for potential confounders, compared to those without PE, SLE patients with $\mathrm{PE}$ had significantly higher inpatient mortality [6\% vs $2.0 \%$, OR $2.99(\mathrm{p}<0.001)]$, greater disability at discharge $[31 \%$ vs $26 \%$, OR $1.26(\mathrm{p}<0.001)]$, longer length of stay(LOS) by 2.91 days and higher cost of hospitalisation by \$19400 [table 1].

In comparison to 10 -year-analysis of National Hospital Discharge Survey (NHDS) database, the results show similar overall increase in risk of developing PE with SLE, mean age, sex ratio, length of stay, higher risk in African-Americans and increased mortality except, in NIS database, PE was more common in males not females (table 2).

Conclusion SLE significantly increases the risk of developing $\mathrm{PE}$ in hospitalised patients. Furthermore, PE with SLE is associated with significantly higher mortality and cost of hospitalisation, increased LOS and greater disability at discharge. These results also suggest that African-Americans may have a higher risk of PE but role of sex needs further evaluation. These results suggest thromboembolism prophylaxis should be considered in hospitalised SLE patients but more studies are needed to further elucidate the relationship and risk of PE in SLE, especially in hospitalised patients.

\section{PS3:48 THE INCIDENCE OF CARDIOVASCULAR EVENTS IN ITALIAN PATIENTS WITH SYSTEMIC LUPUS ERYTHEMATOSUS IS LOWER THAN IN NORTH EUROPEAN AND AMERICAN COHORTS: IMPLICATION OF DISEASE-ASSOCIATED AND TRADITIONAL RIS}

${ }^{1} S$ Fasano, ${ }^{2} D P$ Margiotta, ${ }^{3} R$ Gualtierotti, ${ }^{4} A$ Corrado, ${ }^{5} \mathrm{O}$ Berardicurti, ${ }^{1} L$ Pierro, ${ }^{1} \mathrm{~A}$ Riccardi, ${ }^{5} \mathrm{R}$ Giacomelli, ${ }^{4} \mathrm{FP}$ Cantatore, ${ }^{3} \mathrm{P}$ Meroni, ${ }^{2} \mathrm{~A}$ Afeltra, ${ }^{1} \mathrm{G}$ Valentini. ${ }^{1}$ Division of Rheumatology, Department of Clinical and Experimental Medicine, University of Campania Luigi Vanvitelli, Naples, Italy; ${ }^{2}$ Clinical Medicine and Rheumatology Department, Campus Bio-Medico University of Rome, Italy; ${ }^{3}$ Division of Rheumatology, ASST Pini, Department of Clinical Sciences and Community Health, University of Milan, Italy; ${ }^{4}$ Rheumatology Clinic, Department of Medical and Surgical Sciences, University of Foggia Medical School, Foggia, Italy; ${ }^{5}$ Division of Rheumatology, Department of Biotechnological and Applied Clinical Science, University of L'Aquila, Italy

\subsection{6/lupus-2018-abstract.96}

Background Previous study from our group have pointed out a lower number of cardiovascular (CV) events in Italian patients with Systemic Lupus Erythematosus (SLE) than in North European and American ones. This study aims to assess the incidence of the first CV event in a large, multicenter, Italian cohort of patients with SLE and search for differences in disease and traditional risk factors among distinct cohorts. Methods Clinical charts of SLE patients consecutively admitted to five Italian rheumatologic centres from November 1 st 2000 and December 31 st 2015 were retrospectively studied. Patients selected were free of $\mathrm{CV}$ events at baseline. $\mathrm{CV}$ incidence rate was expressed as the number of events in the cohort divided by the total number of years at risk. CV cumulative incidence were evaluated as the proportion of patients who experienced a new CV event over the follow up period. Our incidence was compared with that detected in the Italian general population and those reported in SLE cohorts from other countries.

Results The median duration of follow-up was 6 years $(\mathrm{IQR}=3-11)$. During the observational period, 37(cumulative incidence $=7.2 \%$ ) patients had a first episode of $\mathrm{CV}$ event with an incidence rate of 10.1/1000 person-years i.e 12 times higher than in the general population. The $\mathrm{CV}$ incidence rate and cumulative incidence detected in our Italian cohort was lower than those from North European and American cohorts. The Italian cohort differed from other SLE cohorts in some 